\title{
The Comparison on the Side Feed Mode of Micro-strip Patch
}

\author{
Wen Dang \\ Department of Communication and Information \\ Xi'an Technology University \\ Lane 78, Yanta Road, \\ Beilin District, Xian, China \\ e-mail: 13637863650@163.com
}

\author{
Xinliang Liu \\ Department of Communication and Information \\ Xi'an Technology University \\ Lane 78, Yanta Road, \\ Beilin District, Xian, China \\ e-mail: 84829562@qq.com
}

\begin{abstract}
The feeding of the micro-strip patch is flexible and complex, especially side feeding. In this paper, a single microstrip patch antenna with a center frequency of $2.45 \mathrm{GHZ}$ is taken as an example to explore the performance of the slotted feed and non-slotted feed and verified in HFSS15.0. Combined with HFSS15.0 software simulation and theoretical calculation, the corresponding conclusions can be obtained. The theoretical value and simulation results will show that: the required 1/4 converter sizes of the non-slotted side feed and slotted side feed are different. By contrasting with sizes of two feeding modes, electrical size of the slotted feed is smaller. It can be known that all performances of the slotted feed are more superior.
\end{abstract}

Keywords-HFSS Simulation; Micro-strip Patch; Side-feed; Slot; Matching

\section{INTRODUCTION}

Antenna is a necessary component for radiating and receiving radio waves in engineering systems such as wireless communications, radio and television, navigation, satellite, radar and other engineering systems. The microstrip patch is simple and easy to plastic, its feeding modes are flexible and changeable. The stability of the antenna can be also affected by micro-strip patch feeding mode. The different feeding mode has different properties. The design of the feeding system determines the current distribution of the radiation patch. The more consistent the current direction is, the higher the gain is, the greater the energy is, and the better the directivity is [3]. Therefore, in an antenna system, it is especially important that how the feeding system should design, which determines the stability of the system and the quality of microwave devices.

In this paper, two different side feed modes of the radiation patch are analyzed and compared. By simulating the cell structure of the micro-strip patch antenna with the center frequency $\mathrm{f}$ at $2.45 \mathrm{GHZ}$ in HFSS15.0, the corresponding sizes of the 1/4 impedance converter and intrinsic impedance are different, when the feeding position is not the same place, which has been validated in HFSS15.0. The mode following the theoretical sizes calculated by given equations is simulated in HFSS15.0. The simulation results have been drawn the followings. For the non-slotted side feed, the radiation impedance is caused by the edge impedance of the patch. The theoretical calculated 1/4 converter has a large error between the length and width values. To achieve the minimum S11 at the center frequency, it is necessary to optimize the micro-strip line parameters for several times. For the slotted side feed, the radiation impedance is produced by the edge impedance of the patch and the edge impedance of the groove. The theoretical value of the 1/4 converter has a higher accuracy. The best performance of the center frequency can be achieved by adjusting the width and depth of the slotted feeding. Based on the above conditions, the minimum value of the antenna S11 can be obtained, the smaller the reflection energy is, the greater the transmission efficiency is.

\section{DESIGN AND ANALYSIS OF RADIATION PATCH UNIT}

In this paper, the radiation patch of the center frequency $\mathrm{f}$ $=2.45 \mathrm{GHZ}$ would be used as an example, the thickness $\mathrm{h}$ of the substrate with Rogers 5880 is $1.5 \mathrm{~mm}$, its dielectric constant is 2.2. According to the formula (1) - (5) [8] - [9] , it can be obtained that the width $\mathrm{w}$, length $\mathrm{L}$ were $40 \mathrm{~mm}$ and $48.4 \mathrm{~mm}$ respectively, as well as the input impedance.

The width $\mathrm{w}$ of radiation patch is the following:

$$
w=\frac{c}{2 f}\left(\frac{\varepsilon_{r}+1}{2}\right)^{-\frac{1}{2}}
$$

Where $\mathrm{c}$ is the speed of free space wave

Taking into account the edge shortening effect, the actual length $\mathrm{L}$ of the radiation patch should be expressed as:

$$
L=\frac{c}{2 f \sqrt{\varepsilon_{e}}}-2 \Delta L
$$

$\varepsilon_{\mathrm{e}}$

Where is the effective permittivity, $\Delta L$ is the length of the equivalent radiation gap, they can be calculated by using the following formulas respectively.

$$
\varepsilon_{\mathrm{e}}=\frac{\varepsilon_{r}+1}{2}+\frac{\varepsilon_{r}-1}{2}\left(1+12 \frac{h}{w}\right)^{-\frac{1}{2}}
$$




$$
\Delta L=0.412 h \frac{\left(\varepsilon_{\mathrm{e}}+0.3\right)(w / h+0.264)}{\left(\varepsilon_{e}-0.258\right)(w / h+0.8)}
$$

The input impedance can be obtained as following:

$$
Y_{\text {in }}(z)=\frac{2 G}{\cos ^{2}(\beta z)}
$$

Where $\mathrm{G}$ is the radiation conductance, $\beta$ is the phase constant in the medium, and $\mathrm{z}$ is the distance from the feed point to the radiation patch edge. It can be concluded that the different input impedance can be obtained by selecting different feeding point positions.

\section{A. Radiation Patch Unit}

The length and width of the radiation patch can be calculated by formulas (1)-(4). The edge impedance of the radiation patch can be obtained by formula (5). The length and width of the $1 / 4$ impendence converter can be known by the edge impedance. The width and length of the $50 \Omega$ intrinsic impedance can be also counted. According to the optimum sizes, the model can be built in hfss 15.0 shown in Fig.1.

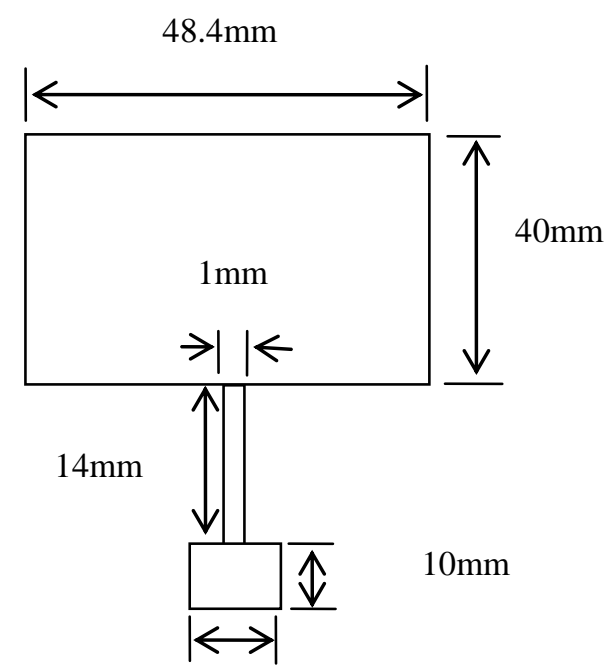

$6 \mathrm{~mm}$

Figure 1. No slotted patch antenna

The size of the slotted micro-strip patch can be also calculated by the same method. The optimum sizes can be obtained after many optimizations, it can be built in hfss 15.0 shown in Fig.2.

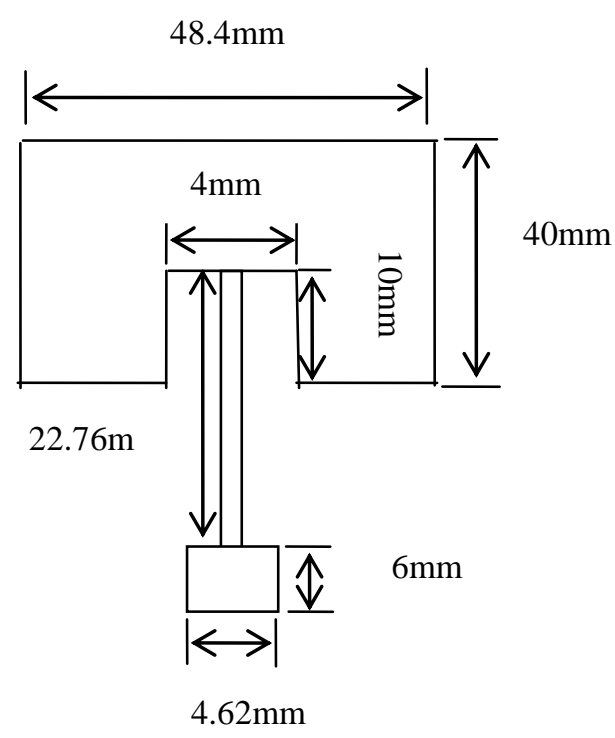

Figure 2. Slotted patch antenna

After hfss15.0 simulation, the results are obtained in Table I . The simulation results obtained by the two feeding methods are basically the same. The theoretical sizes of the slotted feed is closer to the simulated ones, even almost identical, and its electrical size is smaller. When optimizing in the way of the slotted feed, the workloads can be greatly reduced and the efficiency can be improved.

\section{B. Verification}

In order to verify the rationality of the above conclusions, the input port can be excited by the wave port with a $50 \Omega$ load. when the width of the micro-strip line is w0 and the medium thickness is $\mathrm{h}$, the height of the wave port is generally set to $6 \sim 10 \mathrm{~h}$. When there is $\mathrm{w} 0>\mathrm{h}$, the width of the wave port is set to $10 \mathrm{w} 0$. When there is $\mathrm{w} 0<\mathrm{h}$, the width of the wave port is generally set to $5 \mathrm{w} 0$ or $3 \sim 4 \mathrm{~h}[8]$. According to the rules, it can be built and verified in HFSS15.0. The size and impedance of each port are shown in Table I .

From Table I, it can be seen that the impedance value can be directly gotten by adding wave-port excitation for the converter as the input impedance, after running in hfss15.0. The slotted feed mode is closer to the theoretical value than the non-slotted feed mode. Thus, the slotted feed mode can improve the efficiency of the operation and reduce the workload.

\section{Comparison and New Discoveries of Two Feed Modes}

In the micro-strip feed, the return loss can be affected by the size of the $1 / 4$ converter. The reflected voltage wave in the circuit can be reduced by proper using the $1 / 4$ converter, the loss can be also reduced. The different performances of slotted and non-slotted feed are shown in Table I . 
TABLE I. COMPARISON BETWEEN THE SLOTTED AND NON-SLOTTED FEEDING ( UNIT: MM )

\begin{tabular}{|c|c|c|c|c|}
\hline & & The Slotted & The Non-slotted & \\
\hline & & Optimization & Optimization & Calculation \\
\hline \multicolumn{2}{|c|}{ The length of patch } & 48.4 & 48.4 & 48.40 \\
\hline \multicolumn{2}{|c|}{ The wide of patch } & 40 & 40 & 39.70 \\
\hline \multirow{3}{*}{ The port of $1 / 4$ converter } & length & 22.76 & 14 & 22.76 \\
\hline & wide & 2.1 & 1 & 2.14 \\
\hline & impedance & $82.3 \Omega$ & $116.2 \Omega$ & $79.37 \Omega$ \\
\hline \multirow{3}{*}{ The port of $50 \Omega$} & length & 4.62 & 6 & $4.62 \Omega$ \\
\hline & wide & 6 & 10 & \\
\hline & impedance & $50.7 \Omega$ & $42.2 \Omega$ & $50 \Omega$ \\
\hline \multicolumn{2}{|c|}{ Substrate thickness $\mathrm{H}$} & 1.5 & 1.5 & 1.5 \\
\hline \multicolumn{2}{|c|}{ The wide of the slotted } & 4 & & \\
\hline \multicolumn{2}{|c|}{ The length of the slotted } & 10 & & \\
\hline \multicolumn{2}{|c|}{$\mathrm{S} 11$} & $-45.3 \mathrm{db}$ & $-37.9 \mathrm{db}$ & \\
\hline \multicolumn{2}{|l|}{ gain } & $7.89 \mathrm{db}$ & $7.82 \mathrm{db}$ & \\
\hline \multicolumn{2}{|l|}{ Size } & $2010 \mathrm{~mm} 2$ & $1971.516 \mathrm{~mm} 2$ & \\
\hline \multicolumn{2}{|c|}{ bandwidth } & $1.2 \%$ & $1.2 \%$ & \\
\hline \multicolumn{2}{|c|}{ reduction } & & $2 \%$ & \\
\hline
\end{tabular}

From Table I, it can be concluded that the electrical size of the slotted feed is slightly smaller than the electrical size of the non-slotted feed, in that case where the return loss, the gain and bandwidth of the same patch are substantially consistent. The theoretical value of the slotted feed size is closer to the optimum value. Therefore, when the micro-strip arrays are formed, the advantages of the slotted feed are more obvious.

\section{CONCLUSION}

According to comparison and analysis of the simulation results of the two feeding methods, the following conclusions can be drawn:

The value of the S11 is closely related with the size of the micro-strip line, especially the width of the micro-strip line. The impact of the width play important role, the center frequency and the ideal value can be coincident by adjusting the width. The value of S11 can be made better by adjusting the length, So that the feed network can achieve full match.

The 1/4 converter ( $Z 1$ ) of the non-slotted feed is determined by the micro-strip characteristic impedance (ZL) and the edge impedance (ZL) of the patch. Similarly, the edge impedance of the slotted feed patch is mainly caused by the edge of the slot and the radiation patch.

The edge impedance and micro-strip line impedance can be calculated in HFSS15.0 by directly adding wave port excitation. After calculation, the impedance $\mathrm{Z} 0$ values can be gotten as shown in Table I .

In short, all performances of the slotted feed are better than ones of the non-slotted feed. In the feed mode selection, the slotted feed is a relatively stable feeding method.

\section{The DeVElopment Prospect AND SignificANCE}

In the paper, by comparing the performances of two different feeding methods[11], it could been seen that performances of all aspects of slotted feed are even better. When the slotted feed is matched, the theoretical value is more accurate without too much optimization, saving a lot of time and improving the simulation efficiency, which provides a good foundation for the micros-trip antenna array. The slotted radiation patch reduces the electrical size of the patch greatly, so that the antenna structure is more compact and miniaturization. This will be the future development tendency of micro-strip antennas.

Today, micro-strip theory is mature and widely used. With the wide application and great development of micro-strip antenna in the fields of satellite communication [5], millimeter-wave [4] and mobile communication, the research of micro-strip planar antenna technology [10] will become one of the hotspots in future antenna research. At present, the compact profile of the antenna is a welcomed developed tendency [6] - [7]. The sizes of the radiation patch, whether it is as a unit patch micro-strip antenna, or as the array antenna unit array, affect the antenna resonance point and other performances. Stable basic unit structure will be provided broadband for the micro-strip patch as good foundation.

\section{ACKNOWLEDGEMENT}

I would like to thank all my teachers who have helped me to develop the fundamental and essential academic competence. My sincere appreciation also goes to all the teachers and students who helped me. 


\section{REFERENCES}

[1] S. L. S. Yang, K. F. Lee, and A. A. Kishk, " Design and study of wideband signal feed circularity polarized microstrip antenna," Progress In Electromagnetics Research, PIER 80, 45-61, 2008

[2] Huynh, T. and K. F. Lee, "Single-layer single-patch wideband microstrip antenna," Electron. Lett., Vol. 31, No. 16, 1310-1312, Aug. 1995.

[3] Bhalla, R. and L. Shafai, "Resonance behavior of single U-slot microstrip patch Antenna," Microwave and Optical Technology Letters, Vol. 32, No. 5, 333-335, March 2002.

[4] Z. Pi and F. Khan, "An introduction to millimeter-wave mobile broadband systems," IEEE Commun. Mag., vol. 49, no. 6, pp. 101-107, Jun. 2011.

[5] E. Arnieri, L. Boccia, G. Amendola, and G. Di Massa, "A compact high gain antenna for small satellite applications," IEEE Trans. Antennas Propag., vol. 55, no. 2, pp. 277-282, Feb. 2007.
[6] K. L. Wong, Compact and Broadband Microstrip Antennas. Hoboken, NJ: Wiley, 2002.

[7] S. K. Podilchak, M. Caillet, D. Lee, Y. M. M. Antar, L. Chu, M. Hammar, D. Caldwell, and E. Barron, "A compact circularly polarized antenna using anarray of folded-shorted patches," IEEE Trans. Antennas Propagation, vol. 61, no. 9, pp. 4861-4867, Sept. 2013.

[8] Li Mingyang. Liu Yang.Design of HFSS Antenna [M]. Beijing: Electronic Industry Press, 2014.

[9] John Klaus• Antenna (Third Edition, Volume 1) [M]. Beijing: Electronic Industry Press, 2004.

[10] Deng Weibo. Analysis of characteristics of high frequency monopole antenna on dielectric plane [J]. Journal of Electronics \& Information Technology, 2001,23 (12).

[11] Li Xiangqiang. Liu Qingxiang. Zhao Liu.et. 3-ring 48-element high power radial line helical array antenna. High Power Laser and Partiacl Beams. 2006.18(5):843-846. 